\title{
Pleural ultrasonography. Pictorial essay.
}

\author{
Romeo Chira ${ }^{1}$, Alexandra Chira ${ }^{2}$, Roberta Mânzat Săplăcan ${ }^{1}$, Georgiana Nagy ${ }^{1}$, \\ Adriana Bințințan'1, Petru Adrian Mircea ${ }^{1}$
}

${ }_{1}^{1} 1^{\text {st }}$ Medical Clinic, ${ }^{2} 2^{\text {nd }}$ Medical Clinic, "Iuliu Hatieganu” University of Medicine and Pharmacy, Cluj-Napoca, Romania

\begin{abstract}
Pleural ultrasonography (US) represents nowadays a useful diagnostic tool in the management of pleural diseases. Detection and evaluation of pleural effusions, pneumothorax and pleural-based lesions can be performed with US, which has the advantages of wide availability, low cost, absence of radiation exposure, and portability. It is the best method for guiding interventional procedures in the pleural space. It has also limitations, due to the interposition of bony parts of the thorax, subcutaneous emphysema and inability to visualize the mediastinal pleura. US of the thorax is an operator dependent technique demanding an experienced operator in order to obtain reliable results. This pictorial essay presents various ultrasound findings of the diseases which concern the pleura.
\end{abstract}

Keywords: ultrasonography, pleural diseases, lung diseases.

\section{Introduction}

Progresses realized by ultrasound examination in last decays placed this imaging method aside computer tomography $(\mathrm{CT})$ and magnetic resonance imaging in the medical management of the pleuropulmonary diseases.

Classic advantages of the ultrasonography (US) wide availability, lower cost, possibility of bedside examination, shorter duration of examination comparing with $\mathrm{CT}$, and possibility of guiding different invasive procedures - are now doubled by contrast enhanced US examination, different modes of elastography, and a better technology which have improved the grayscale image.

Pleural ultrasonography has widespread indications, being able to help clinicians in many situations - either in emergencies and bedside conditions, or in differential diagnosis of doubtful radiological images [1-4].

Received 20.09.2014 Accepted 2.10.2014

Med Ultrason

2014, Vol. 16, No 4, 364-371

Corresponding author: Romeo Chira, $\mathrm{MD}, \mathrm{PhD}$

$1^{\text {st }}$ Medical Clinic, Dept. Gastroenterology Emergency Clinical County Hospital, Cluj

"Iuliu Hatieganu" University of Medicine and Pharmacy

3-5 Clinicilor, 400006, Cluj-Napoca, Romania

Phone: 0040264592771/1172

Email: romeochira@yahoo.com

\section{Technique of pleural US}

Examination of the pleura is part of the evaluation of the thoracic wall and surface of the lungs. It is recommended that the physician who performs the US examination has access to the relevant clinical data and, most importantly, to the previous results of other imagistic examinations. This information is important for the US procedure, to shorten the time of the examination, knowing that only about $70 \%$ of the pleural surface can be evaluated with ultrasound in the absence of voluminous pleural effusions [4]. In the latter cases, also mediastinal pleura can be visualized, in various proportions.

For transthoracic examination of the pleura it is common to use two types of transducers. First, a lower frequency transducer $-3-5 \mathrm{MHz}$ is used, which has a wider and deeper image field, allowing easier detection of the anomalies of intrathoracic structures. Examination of the pleura follows the surface of the thorax corresponding to the soft structures - supraclavicular and intercostal spaces - using sagittal and transversal sections initially. Due to the obliquity of the intercostal spaces oblique and coronal sections, and commonly transabdominal - subcostal recurrent or sagittal sections are used, offering a transhepatic and transsplenic approach to the diaphragmatic pleura. After a pathological process is detected, using a higher frequency trans- 


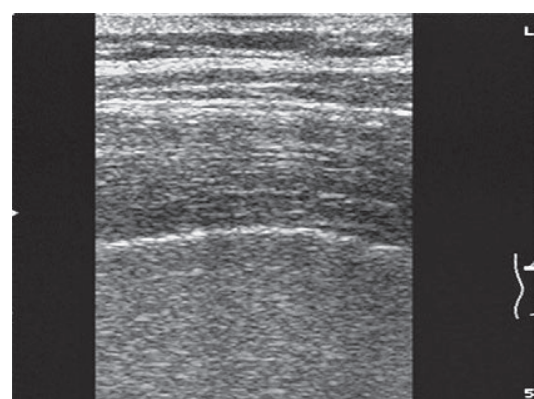

Fig 1. Stratified structure of the thoracic wall and hyperechoic pleuropulmonary interface, without separation of the pleural sheets; examination with $7.5 \mathrm{MHz}$ transducer.

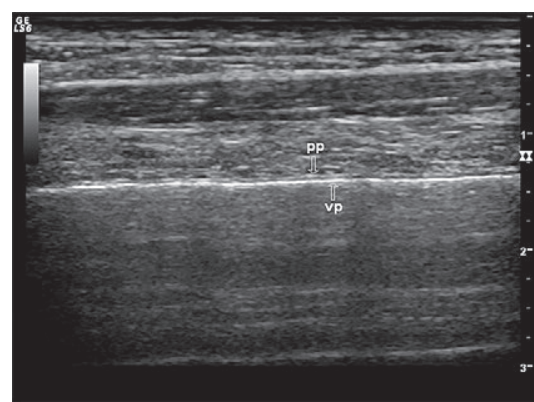

Fig 2. Image of the thoracic wall obtained with high frequency transducer $-13 \mathrm{MHz}$ : identification of the parietal pleura (pp), pleural space and visceral pleura (vp).

ducer -7.5 to $13 \mathrm{MHz}$ - the superficial pathology can be better characterized.

Pleural sheets are visualized at the pleuropulmonary interface, usually indistinguishable from the lung, when a lower frequency transducer is used and in normal conditions (fig 1). When we turn to higher frequencies, the parietal pleura is individualized, a minimal transonic space follows, corresponding to the pleural space, and visceral pleura is added to the hyperechoic interface generated by the air from the lung (fig 2). Normal thickness of the pleura is less than $3 \mathrm{~mm}[4,5]$.

\section{Pleural pathology}

\section{Pleural effusions}

US detection of the pleural effusions is very sensitive, even for minute amounts of fluid, being superior to the standard radiological methods [6]. It is more accurate for the quantification of pleural effusion than chest radiography $[7,8]$. A pleural effusion appears as an anechoic space (usually), homogeneous, between pleural sheets (fig 3). Atelectatic lung is visible as a tongue-like structure within the collection (fig 4), which expands partially

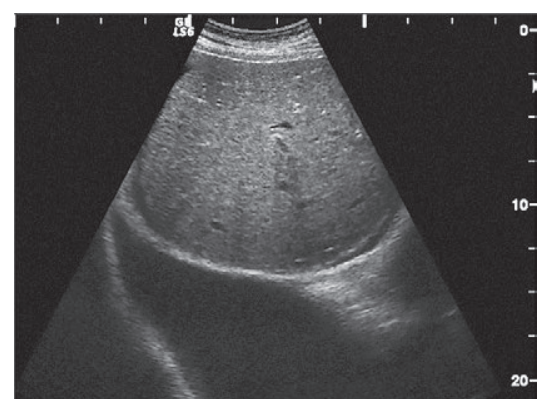

Fig 3. Large pleural effusion on the right side, visualized using transhepatic approach.

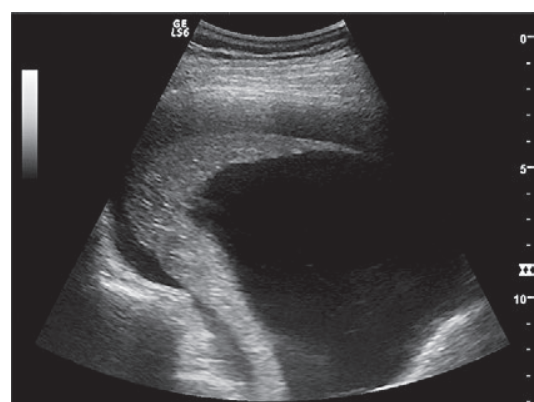

Fig 4. Large effusion containing the "tongue-like" appearance of atelectatic lung.

with breathing and sometimes (on the left side mostly), pulses with cardiac systoles. The shape of the effusion also changes with respiratory movements, when loculation is absent. When adhesions between pleural sheets are present, changes in shape and lung motions are also absent.

Diagnostic of medium or large pleural collections is relatively easy, but small amounts of fluid, or loculated, or cases with associated pleural thickening can raise difficulties. One particular useful sign for depiction of liquid is "fluid color" sign, a color signal visible in color Doppler mode within the pleural collection, during cardiac cycles or respiratory movements. The sensibility of the fluid color sign for the detection small amounts of fluid or loculated collection is $89.2 \%$, and the specificity is $100 \%$ [3].

Classification of the pleural effusions is based on echogenicity and structure and includes four types: anechoic, complex non-septated, complex septated and homogenously echogenic [9]. Transudative effusions appear as anechoic, non-septated spaces, and free flowing. Anechoic effusion can be also exsudative, not only transudates. Exudative effusions contain proteins, fibrin, 
inflammatory cells, blood cells and may appear as complex non-septated, echogenic or complex septated $[4,9]$. Inflammatory effusions present echogenic strands or septations, usually mobile with respiration and cardiac activity (fig 5). When pleural nodules or pleural thickening are present, they are associated with exudates. Homogeneously echogenic effusions are realized by hemothorax, chylothorax or epyema (fig 6) [4,9]. Empyema is one of the most important type of complications in the evolution of pneumonia, chest trauma, surgery and esophageal rupture. It has three stages, with different aspects. In the first, the exudative stage appears as an inflammatory (strands,

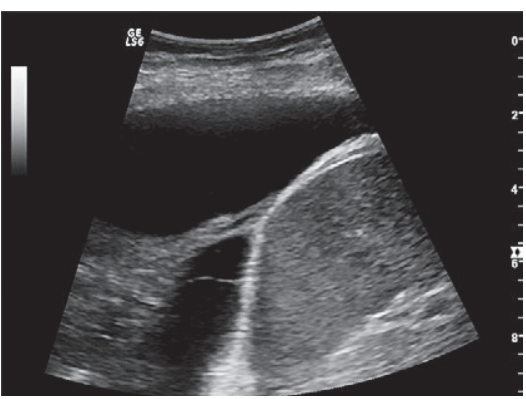

Fig 5. Inflammatory complex septated pleural effusion with adhesions between lung and diaphragm.

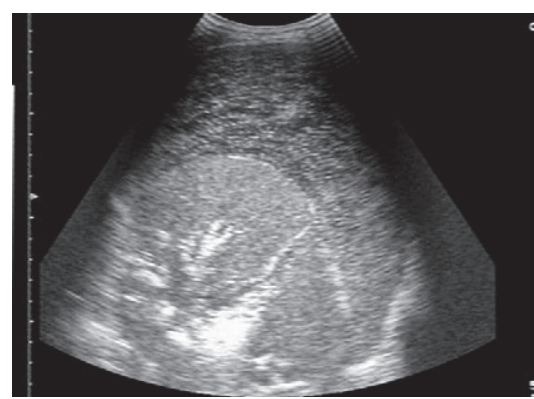

Fig 6. Echogenic pleural effusion hemothorax, with atelectactic lung presenting aeric bronchograms.

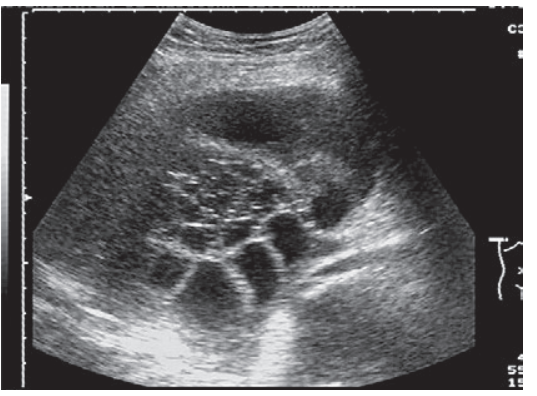

Fig 7. Exudative stage of empyema multiloculated pleural effusion. echogenic floating echoes) or a multiloculated effusion where various echogenicity may be seen (fig 7). In the second, fibrinopurulent stage, thickening of the pleura occurs, due to the deposition of fibrin (fig 8). The organized stage is characterized by the formation of a rigid thick membrane called pleural peel around the lung (fig 9) [10]. Malignant effusions are usually large and may present floating echoes and sometimes metastatic nodules (fig 10) [11]. Commonly, the size of the nodules is small - less than 1-2 $\mathrm{mm}$ - and they cannot be detected by US [11].

Pneumothorax can be quickly and reliably diagnosed with ultrasonography, even at the bedside, with a greater

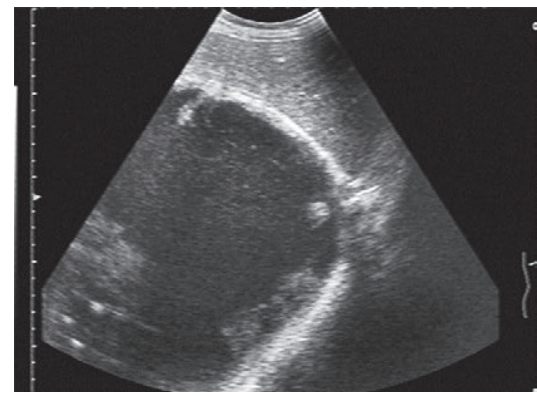

Fig 8. Fibrinopurulent stage of empyema - thick-wall collection with echogenic debris.

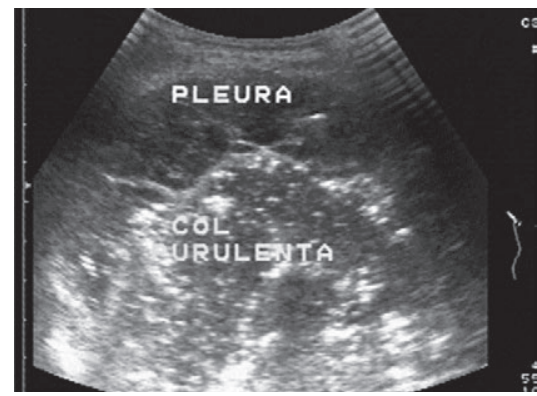

Fig 9. Organized stage of empyema very thick wall (pleural peel) and inhomogeneous effusion containing large debris.

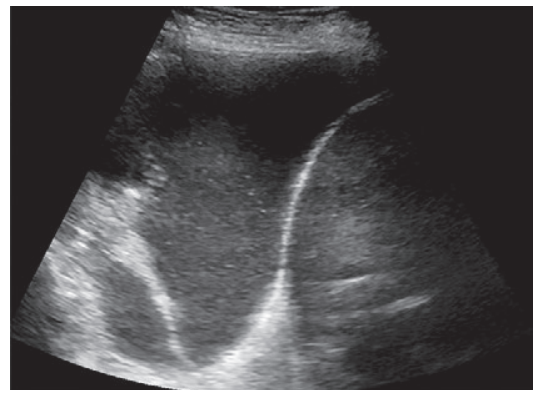

Fig 10. Large malignant effusion with echogenic floating particles and atelectatic lung. 
sensitivity than chest radiography [12]. There are B mode signs and also an $\mathrm{M}$ mode sign. The classical ultrasonographical signs of pneumothorax are absence of lung sliding, of B lines and lung pulse, and presence of lung point [13-16]. In M mode normal aspect of "sea shore" corresponding to peripheral aerated lung is replaced with the "stratosphere" sign where the pneumothorax is present $[17,18]$.

Hydropneumothorax is more difficult to recognize, but sometimes the clinical context is helpful - recent thoracocentesis or chest trauma, history of pneumothoraces. Pleural effusion is present in various amounts,

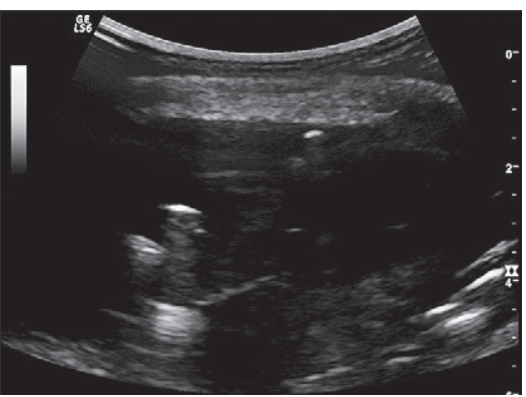

Fig 11. Hydropneumothorax - pleural effusion containing isolated small hyperechogenic imagines.

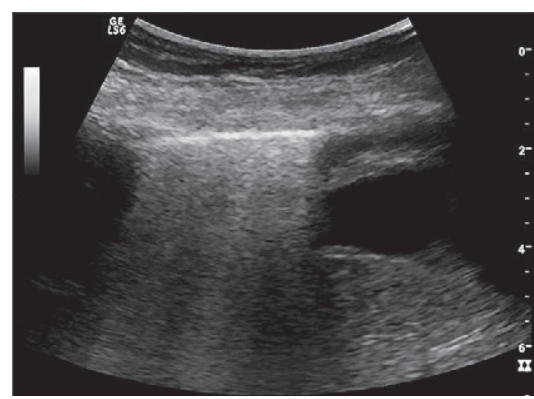

Fig 12. Large hydropneumothorax - with free air floating in a large effusion.

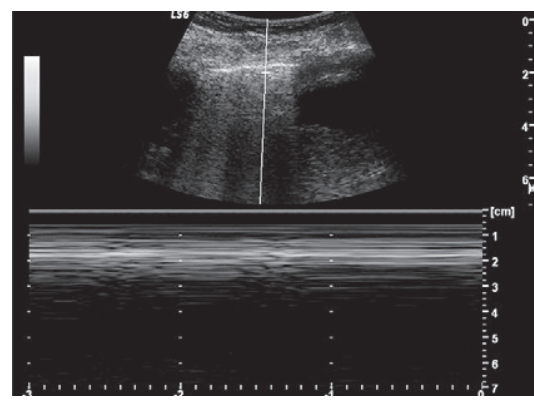

Fig 13. Stratosphere sign corresponding to air inside a pleural effusion. and contains hyperechoic isolated floating images, corresponding to air bubbles (fig 11). Sometimes it appears as linear hyperechoic images situated under the thoracic wall, surrounded by effusion, with the pathognomonic stratosphere sign (fig 12, fig 13).

\section{Pleural thickening}

Pleural thickening can be diffuse or focal. Diffuse type can be caused by pleural fibrosis or infiltration and metastatic disease. Fibrosis affects visceral pleura, followed by restriction of lung ventilation. Pleural plaques appear as hypoechoic ellipsoidal lesions more than $2 \mathrm{~mm}$ thick (fig 14, fig 15). If the disease is recent, the plaques

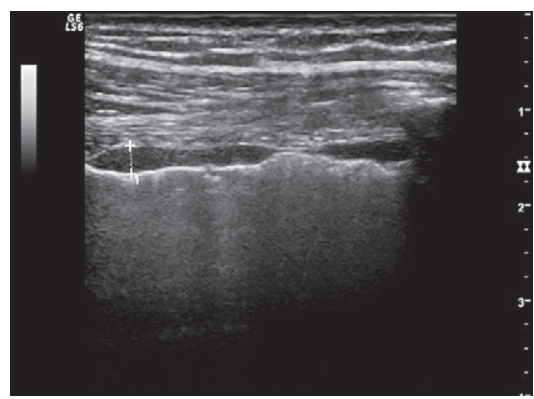

Fig 14. Two pleural plaques - ovoid hypoechoic homogeneous lesions.

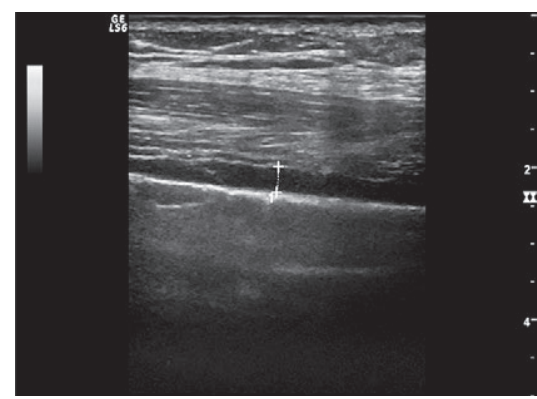

Fig 15. Pleural thickening - a larger hypoechoic, homogeneous pleural plaque.

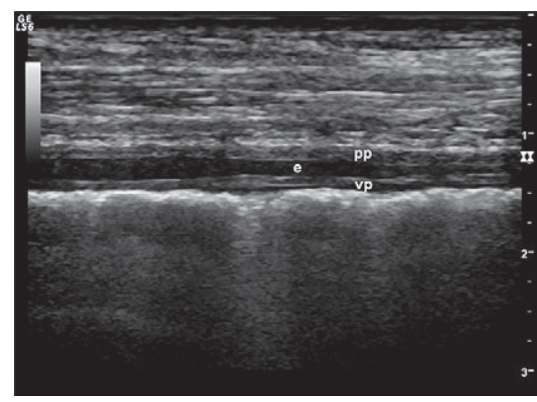

Fig 16. Postpneumonic pleural thickening - both sheets are mildly thickened ( $\mathrm{pp}$ - parietal pleura, vp - visceral pleura) with a minute amount of pleural fluid. 


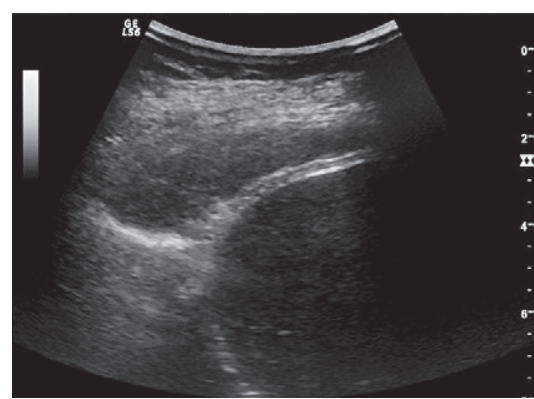

Fig 17. Markedly enlarged pleura at right anterior costophrenic sinus with sharp edges, homogeneous structure, hypoechoic - postazbestozic plaque.

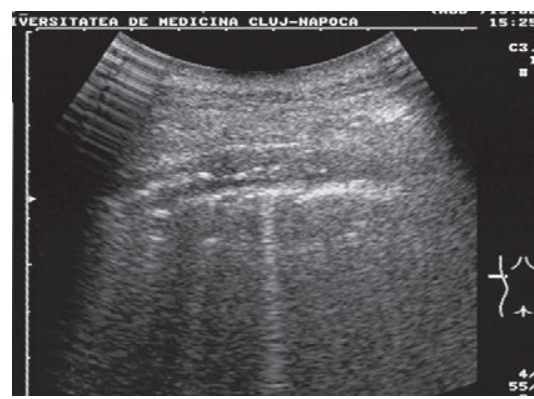

Fig 18. Pleural thickening with hyperechoic deposits and acoustic shadows which obscure partially the lung parenchyma - calcified pachypleuritis

that mimic a loculated effusion may be very hypoechoic. They are usually hypovascularized in color Doppler mode, and the fluid color sign is negative in this situation. Benign pleural thickening is secondary to lung diseases (pneumonia, infarction), asbestosis, trauma, pleurodesis (fig 16, fig 17). Pleural calcifications can occur as a sequel of tuberculosis or empyema [2,3]. In this situation pleural thickening is associated with hyperechoic deposits and acoustic shadows which obscure the lung parenchyma making sometimes the visualisation of deeper lesions impossible (fig 18).

Another cause of pleural thickening is pleuritis. Ultrasonographic aspects include irregular shape of the pleural sheets with pleural discontinuity (89.4\%) (fig 19), small size subpleural consolidation (less than $2 \mathrm{~cm}$ ) $(63.8 \%)$ and parietal or basal pleural effusion $(63.8 \%)$ (fig 20 ) [19].

One of the normal structures which can mimic a pleural thickening is subpleural fat, present in different amounts.

\section{Pleural tumors}

Benign tumors are relatively rare $5 \%$ of pleural tumors), most frequently being represented by a solitary

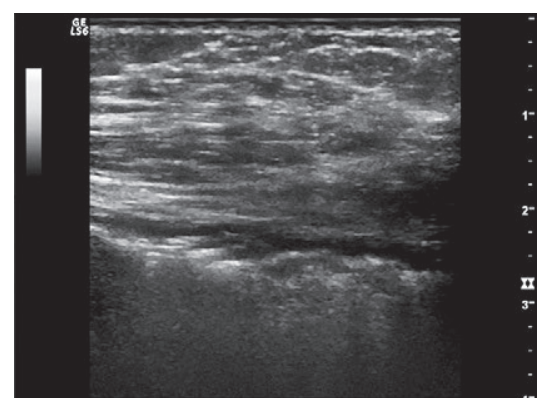

Fig 19. Pleuritis presenting irregular shape of the pleural sheets with pleural discontinuity.

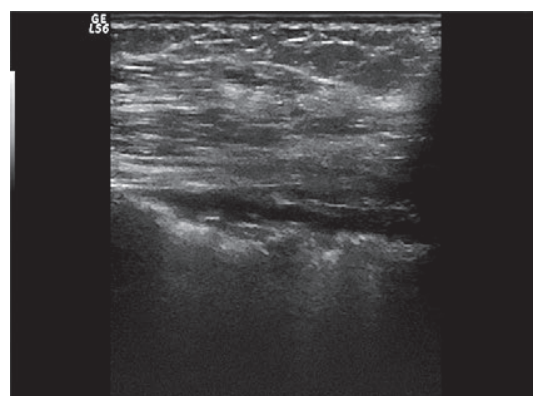

Fig 20. Pleuritis - small pleural effusion accompanying small subpleural consolidations and irregularities of the visceral pleura.

fibrous tumor, lipoma, chondroma, or neurinoma [3]. A solitary fibrous tumor of pleura has a nodular shape, well-defined surface, hypoechoic aspect, and forms an obtuse angle with the chest wall. The lung usually preserves the mobility against the lesion, which can present the vascularization in color Doppler mode (fig 21). The most difficult differential diagnostic is raised by the tumors of the chest wall (for example tumors of the peripheral nerves), which can mimic a parietal pleural mass, and in some cases pathological confirmation is needed. Another diagnostic problem is raised by subpleural hematoma which can present with the aspect of the benign tumor, but clinical context and absence of vascularization are very useful arguments [20].

Malignant primary tumors of the pleura are rare, except for malignant mesothelioma. In these cases, pleural thickening exceeds usually $10 \mathrm{~mm}$ (fig 22), appears focal $(25 \%)$ (fig 23 ) or diffuse (86\%), associated with pleural effusion (74\%) (fig 24) [10]. The lung is irregular and the tumor progresses invading the thoracic wall, lung, contralateral pleura, pericardium, or diaphragm. Calcifications are uncommon, being present in only about $20 \%$ of the cases [10]. 


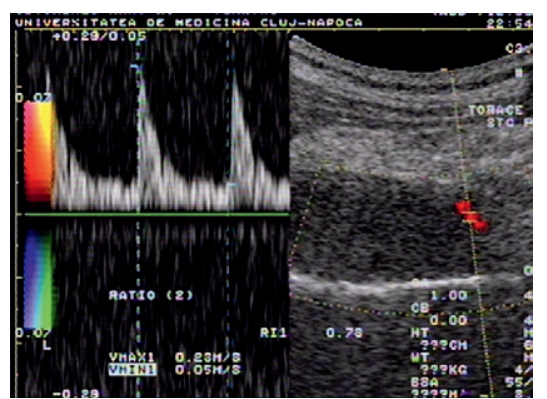

Fig 21. Solitary fibrous tumor of pleura ovoid shape, well-defined surface, hypoechoic and homogeneous structure, presenting arterial supply with color/pulsed Doppler.

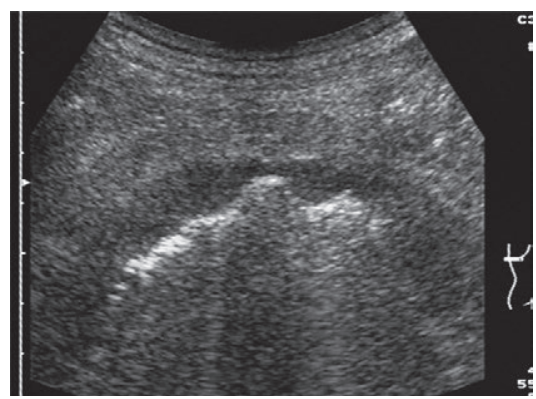

Fig 22. Malignant mesothelioma - pleural thickening more than $10 \mathrm{~mm}$, irregular shape, diffuse type.

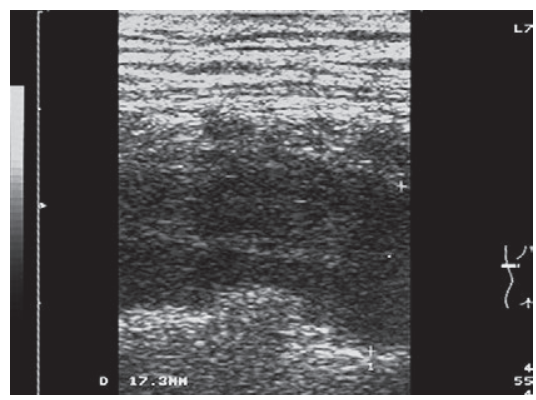

Fig 23. Malignant mesothelioma - pleural thickening more than $10 \mathrm{~mm}$, irregular shape, slightly inhomogeneous.

Pleural metastasis represents the majority of malignant neoplasms of the pleura. The most frequent primary sites of tumors involving the pleura are bronchogenic carcinoma, breast, gastrointestinal tract, kidney and ovaries [4]. US detects multiple nodules with various dimensions (fig 25, fig 26), usually of a small size, accompanied by large effusions (fig 27). The metastatic nodules are hypoechoic, homogeneous, ovoid or irregular shape lesions, showing obtuse angles with the thoracic wall (fig 28). Sometimes the size of the nodules is larger, and the struc-

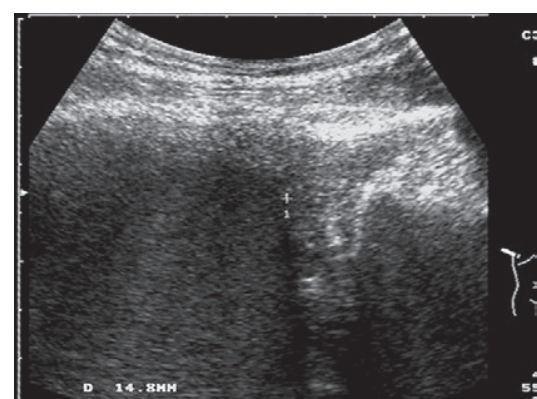

Fig 24. Large echogenic pleural effusion, accompanying markedly thickened parietal pleura $(>10 \mathrm{~mm})$ and diaphragmatic pleura - malignant mesothelioma.

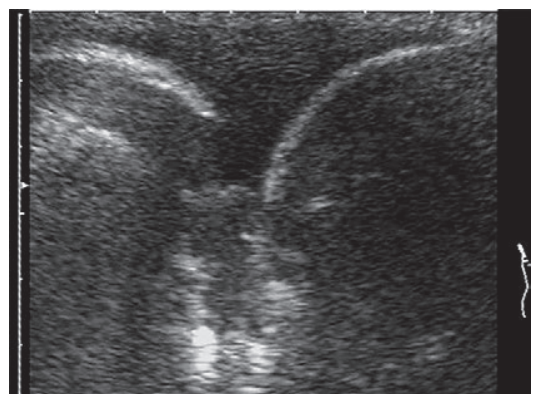

Fig 25. Metastatic nodule of the pleura realizing a symphysis of the inferior lobe of the right lung and diaphragm, with small effusion (primary breast cancer).

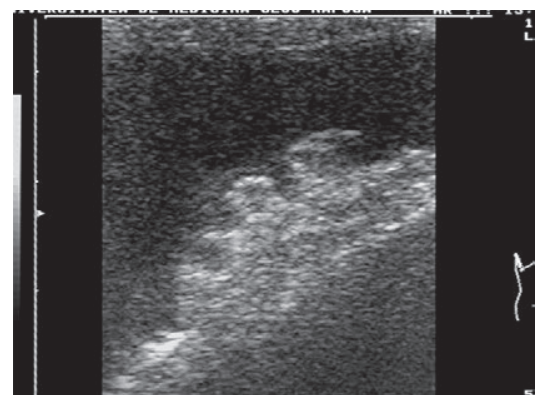

Fig 26. Irregular shape, sessile lesion of the diaphragmatic pleura - metastatic nodules.

ture is inhomogeneous (fig 29). In a patient with known malignancy, a pleural effusion associated with nodules or sheet such as pleural thickening is very suggestive of metastatic disease [2].

The invasion of the pleura, from bronchogenic carcinoma or lung metastasis, appears as hypoechoic lesions abutting the pleura initially with an acute angle to the chest wall. The certain sign of invasion of the parietal pleura is direct visualization of the invasion of the tumor in the structures of the wall or ribs (fig 30). Also, an ob- 


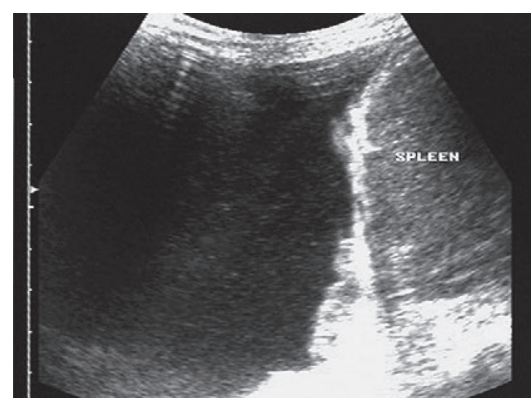

Fig 27. Large left pleural effusion, almost echogenic, with metastatic deposits (adenocarcinoma) on the surface of parietal diaphragmatic pleura.

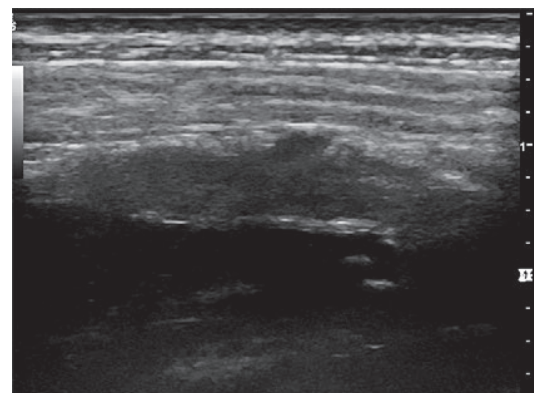

Fig 28. Irregular parietal pleural thickening, associated with effusion - metastatic nodule from squamous bronchogenic carcinoma.

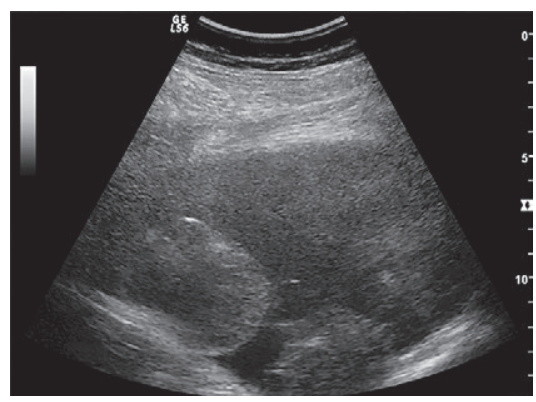

Fig 29. Large metastatic nodules of the pleura from a sarcoma, with voluminous pleural effusion - irregular shape, inhomogeneous lesions.

tuse angle between tumor and the wall, and absence of gliding sign of the tumor with disruption of the pleural lines are suggestive of invasion [21] (fig 31).

\section{Conclusions}

US is a very important diagnostic method for pleural pathology, providing high resolution images, useful

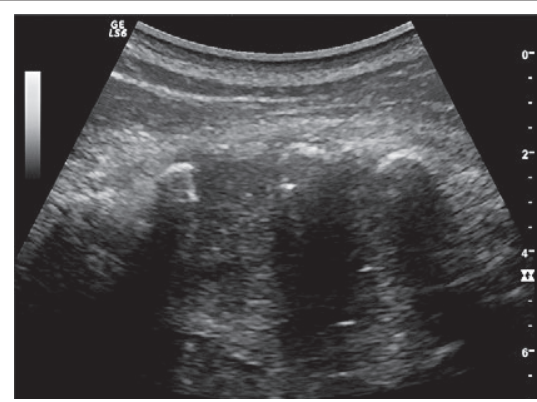

Fig 30. Large bronchogenic carcinoma invading the pleura and the thoracic wall - three ribs are involved.

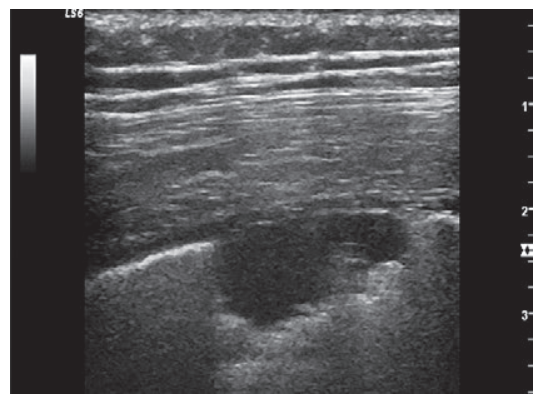

Fig 31. Subpleural lung tumor invading the pleura - disruption of the pleural continuity, obtuse angle between the lesion and the thoracic wall, and absence of the gliding sign in real time.

in all kinds of clinical scenarios - emergency, diagnostic and interventional ultrasonography. It is superior to chest radiography for the diagnosis of pneumothorax and characterization of pleural effusions. US represents the best method for guiding fluid aspiration and transthoracic biopsy of pleural-based lesions. It is also a reliable method for evaluating the parietal infiltration of the lung tumors, but $\mathrm{CT}$ is superior providing more information concerning all parts of the pleura. The limit of this method is the inability to visualize the mediastinal pleura, and the lesions obscured by subcutaneous emphysema and the bony structures of the thorax - scapulae, sternum and the spine.

US can provide useful information for almost all pleural pathology and it should be use more extensively for all these reasons, being also accessible, cheap and widely available for many medical specialties.

\section{Conflict of interest: none}




\section{References}

1. Bollinger CT, Herth FJ, Mayo PH, Miyazawa T, Beamis JF (Eds). Clinical chest ultrasound: from ICU to the bronchoscopy suite. Prog Respir Res. Basel, Karger, 2009; 37: 22-33.

2. Beckh S, Bolcskei PL, Lessnau KD. Real-time chest ultrasonography: a comprehensive review for the pulmonologist. Chest 2002; 122: 1759-1773.

3. Reuss J. The pleura. In: Mathis G. (Ed). Chest Sonography. Springer Verlag Berlin-Heidelberg-New York 2008: 24-45.

4. Sartori S, Tombesi P. Emerging roles for transthoracic ultrasonography in pleuropulmonary pathology. World J Radiol 2010; 2: 83-90.

5. Koh DM, Burke S, Davies N, Padley SP. Transthoracic US of the chest: clinical uses and applications. Radiographics 2002; 22: e1.

6. Gryminski J, Krakowka P, Lypacewicz G. The diagnosis of pleural effusion by ultrasonic and radiologic techniques. Chest 1976; 70: 33-37.

7. Eibenberger KL, Dock WI, Ammann ME, Dorffner R, Hörmann MF, Grabenwöger F. Quantification of pleural effusions: sonography versus radiography. Radiology 1994; 191: 681-684.

8. Kelbel C, Borner N, Schadmand S, et al. Diagnosis of pleural effusions and atelectases: sonography and radiology compared. Rofo 1991; 154: 159-163.

9. Yang PC, Luh KT, Chang DB, Wu HD, Yu CJ, Kuo SH. Value of sonography in determining the nature of pleural effusion: analysis of 320 cases. AJR Am J Roentgenol 1992; 159: 29-33.

10. Brant WE. The thorax. In: Rumack CM, Wilson SR, Char- boneau JW. (eds). Diagnostic Ultrasound. Mosby-Year Book, St. Louis 1998: 575-597.

11. Mathis G. Thoraxsonography - Part I: Chest wall and pleura. Ultrasound Med Biol 1997; 23: 1131-1139.

12. Lichtenstein DA, Meziere G, Lascols N, et al. Ultrasound diagnosis of occult pneumothorax. Crit Care Med 2005; 33 : 1231-1238.

13. Wernecke K, Galanski M, Peters PE, Hansen J. Pneumothorax: evaluation by ultrasound--preliminary results. J Thorac Imaging 1987; 2: 76-78.

14. Targhetta R, Bourgeois JM, Chavagneux R, et al. Ultrasonic signs of pneumothorax: preliminary work. J Clin Ultrasound 1993; 21: 245-250.

15. Goodman TR, Traill ZC, Phillips AJ, Berger J, Gleeson FV. Ultrasound detection of pneumothorax. Clin Radiol 1999; 54: 736-739.

16. Garofalo G, Busso M, Perotto F, De Pascale A, Fava C. Ultrasound diagnosis of pneumothorax. Radiol Med 2006; 111: 516-525.

17. Chandra S, Narasimhan M. Pleural ultrasonography. Open Crit Care Med J 2010; 3: 26-32.

18. Lichtenstein DA. Lung ultrasound in the critically ill. Ann Intensive Care 2014; 4: 1.

19. Gehmacher O, Kopf A, Scheier M, Bitschnau R, Wertgen T, Mathis G. Can pleurisy be detected with ultrasound? Ultraschall Med 1997; 18: 214-219.

20. Chira R, Chira A, Mircea PA. Thoracic wall ultrasonography-normal and pathological findings. Pictorial essay. Med Ultrason 2011; 13: 228-233.

21. Bandi V, Lunn W, Ernst A, Eberhardt R, Hoffmann H, Herth FJ. Ultrasound vs. CT in detecting chest wall invasion by tumor: a prospective study. Chest 2008; 133: 881-886. 